\title{
Nonlinear material behaviour of spider silk yields robust webs
}

\author{
Steven W. Cranford ${ }^{l, 2}$, Anna Tarakanova ${ }^{l, 3}$, Nicola Pugno ${ }^{4}$, Markus J. Buehler ${ }^{1,2,5 \dagger}$ \\ ${ }^{1}$ Center for Materials Science and Engineering, Massachusetts Institute of Technology, 77 Massachusetts \\ Ave., Cambridge, MA 02139, USA
}

${ }^{2}$ Laboratory for Atomistic and Molecular Mechanics (LAMM), Department of Civil and Environmental Engineering, Massachusetts Institute of Technology, 77 Massachusetts Ave., Cambridge, MA 02139, USA

${ }^{3}$ Department of Applied and Engineering Physics, Cornell University, Ithaca, NY 14853, USA

${ }^{4}$ Laboratory of Bio-Inspired Nanomechanics "Giuseppe Maria Pugno”, Department of Structural Engineering and Geotechnics, Politecnico di Torino, Corso Ducadegli Abruzzi 24, 10129 Torino, Italy

${ }^{5}$ Center for Computational Engineering, Massachusetts Institute of Technology, 77 Massachusetts Ave., Cambridge, MA 02139, USA

Natural materials are renowned for their exquisite designs that optimize function, as illustrated by the elasticity of blood vessels, the toughness of bone and the protection offered by nacre ${ }^{1,2,3,4,5}$. Particularly intriguing are spider silks, with studies having explored properties ranging from their protein sequence $^{6}$ to the geometry of a web ${ }^{7}$. This highly adapted material system ${ }^{8}$, which is wellknown to meet a spider's many needs, exhibits exemplary mechanical properties ${ }^{9,10,11,12,13,14,15}$. It thus comes as no surprise that there has been much interest in the molecular design underpinning the outstanding performance of silk fibres ${ }^{1,6,10,13,19,20}$, and in the mechanical characteristics of web-like structures $^{16,17,18,21}$. Yet it remains unknown how the mechanical characteristics of spider silk contribute to the integrity and performance of a spider web. Here we report web deformation experiments and simulations that identify the nonlinear response of silk threads to stress-involving softening at a yield point and dramatic stiffening at large strain until failure-as crucial for localizing the load-induced deformation and hence for endowing spider webs with robustness. Control simulations confirm that a nonlinear stress response results in superior resistance to defects compared to linear elastic or elastic-plastic (softening) material behaviour. We further show that under distributed loads, such as exerted by wind, the behaviour of silk under small-deformation is essential in maintaining the web's structural integrity. The superior performance of silk in webs is therefore not merely due to its exceptional ultimate strength and strain, but more importantly arises from the nonlinear response of silk threads to strain and their geometrical arrangement in a web.

While spider silk is employed in a myriad of functions from wrapping prey to lining retreats ${ }^{22,23}$, here we focus on silk's structural role in aerial webs and on how silk's material properties relate to web function. The mechanical behaviour of silk, like that of other biological materials, is determined by the nature of its constituent molecules and their hierarchical assembly into fibres ${ }^{13,19,20,24,25,26}$ (Fig. S1). Spider webs themselves are characterized by a highly organized geometry that optimizes their function ${ }^{7,8,16,17,18}$. To explore the contribution of the material characteristics to web function, we develop a web model with spiral and radial threads based on the geometry commonly found in orb webs ${ }^{1}$. The silk material behaviour is parameterized from atomistic simulations of dragline silk from the species Nephila clavipes (Model A) ${ }^{19,20}$ (Fig. 1a-b) and validated against experiments ${ }^{10}$ (Methods Summary). As properties of silk can vary across evolutionary lineages by over $100 \%^{9,27,28}$ (SI Section S1), we avoid species-specific silk properties and use instead a representative model to reflect the characteristic nonlinear stress-strain behaviour of silk found in a web. The mechanical performance of individual silk threads has been previously investigated ${ }^{10,12,13}$, and is in agreement with our model in terms of tensile deformation behaviour.

As a functional structure for a spider, it is rare to see a perfectly intact web-debris, attack, or unstable anchorage can lead to loss of threads (Fig. 1c, inlay). We assess a web's ability to tolerate defects by 
removing web sections (silk threads) and applying a local load (Fig. 1c). Removal of up to $10 \%$ of threads, at different locations relative to the load, has little impact on the web's response; in fact, the ultimate load capacity increases by 3-10\% with the introduction of defects (Fig. 1c). We observed in all cases that failure is limited to the thread to which the force is applied. Loading of a spiral thread results in relatively isolated web distortion (Fig. 1e), whereas loading of a radial thread (Fig. 1f) results in larger deformation $(\approx 20 \%$ more deflection; $\approx 190 \%$ increase in energy dissipation; Fig. 1d). But in both cases, failure is localized (Fig. 1e-f). A comparative study of loading radial versus spiral threads demonstrates that the web's structural performance is dominated by the properties of the stiffer and stronger radial dragline silk (with the force to break radial threads within the web $\approx 150 \%$ higher), suggesting that the spiral threads play non-structural roles (e.g., capturing prey). Experiments on a garden spider web (Fig. 1e-f) are in qualitative agreement with the simulations: they confirm the prediction that failure is localized when loading either a spiral or a radial thread. Complementing these findings, we use our atomistic silk model ${ }^{19,20}$ to connect the stress states of loaded web sections with molecular deformations in the threads (Fig. 1a). Under loading and immediately prior to failure, most radial threads in the structure exhibit deformation states equivalent to the yield regime (regime II in Fig 1a), where the presence of polymer-like semi-amorphous regions permits entropic unfolding of the silk nanocomposite under relatively low stress ${ }^{19,20,29}$. Once unfolding is complete, the system stiffens as stress is transferred to relatively rigid $\beta$-sheet nanocrystals ${ }^{20}$ (regimes III-IV); it finally fails, at the thread where force is applied, as the applied stress is sufficient to rupture the nanocrystals.

Simulation and experiment both indicate that localized failure is a universal characteristic of spider webs. Unresolved is whether this behaviour is unique to silk-like materials or a result of the web's architecture (e.g., a property of the construction material or of the structural design). We therefore systematically compare the response of webs constructed from three different types of fibres with distinct mechanical behaviour (Fig. 2a, left panels): in addition to fibres with the atomistically derived stress-strain behaviour of dragline silk (Model A), we use idealized engineered fibres that exhibit either linear elastic behaviour (Model A') or elastic-perfectly-plastic behaviour that involves severe softening (plastic yield) (Model A'). In all cases, we load one of the radial threads and assume that the failure stress $(\approx 1,400 \mathrm{MPa})$ and strain $(\approx 67 \%$ ) of silk threads are constant so that any changes in deformation behaviour (Fig. 2a, right panels) and web damage (Fig. 2a, middle panels) will be a direct result of differences in the stress-strain behaviour of the fibres. In the case of a web comprised of natural dragline silk (top panels), all radial threads contribute partially to the resistance to loading; but the fact that the material suddenly softens at the yield point, which immediately reduces the initial modulus $(\approx 1,000 \mathrm{MPa})$ by $\approx 80 \%$, ensures that only the loaded radial thread enters regime III and begins to stiffen before it finally fails. With linear elastic material behaviour (middle panels), the loaded radial thread is still subject to the bulk of the load; but adjacent radial threads bear a higher fraction of the ultimate load, which results in a greater delocalization of damage upon failure. With elastic-perfectly-plastic behaviour (bottom panels), the softening of radial threads enhances the load distribution even more throughout the web and thereby greatly increases the damage zone once failure occurs. The increased contribution of the auxiliary radial threads to load resistance as we move from the natural to linear elastic to elastic-perfectly-plastic behaviour results in $34 \%$ higher maximum strength, but 30\% less displacement at failure (Fig. 2b).

The above simulations using atomistically derived silk properties (Model A) assume that the spiral threads and radial threads are made of dragline silk and behave identically, except for differences arising from their different thread diameters. But in real spider webs, spiral threads are composed of more compliant and extensible viscid silk with a failure strain of $\approx 270 \%$ (for the species Araneus diadematus ${ }^{1}$, as an example). To explore the effect of different silks making up the spiral and radial threads, we introduce empirically parameterized viscid spiral threads ${ }^{1}$ (Model B) and find that the results are only marginally affected (Fig. $2 b)$. We also use a model where we parameterize both spiral and radial threads according to empirical data ${ }^{1}$ (Model C), subject this model to the same loading conditions and systematically compare its performance 
against that of models with linear elastic (Model C') and elastic-perfectly-plastic behaviours (Model C' '). We find similar web responses, in that the web made from natural silk is weaker yet localizes damage near the loaded region (SI Section S5).

To explore global loading responses, we subject the web models to a homogeneously distributed wind load with effective wind speeds up to $70 \mathrm{~m} / \mathrm{s}$ (a threshold at which all models fail). The system-level deflection curves (Fig. 2c) reflect the mechanical behaviour of the radial threads, which ultimately transfer load to the web's anchoring points. While the spiral threads undergo increased deflection and capture more of the wind load due to their larger exposed length, they are effectively pinned to the much stiffer dragline radial threads that limit web deflection (Figs. 2c, SI Section S8). For wind speeds $<10 \mathrm{~m} / \mathrm{s}$ there is little difference between the models (Figs. 2c, SI Section S8) and deflections are $<12 \%$ of the total span of the web. This relative uniform structural rigidity of the web is attributed to the initial stiffness of the dragline silk prior to yield (Fig. 1a). But under higher wind loads, the softening behaviour of dragline silk at moderate deformation results in significant web deflection that is greater than the deflections seen with linear elastic and elastic-perfectly-plastic material behaviour (Fig. 2c). We find yield in the threads occurs at wind speeds exceeding $\approx 5 \mathrm{~m} / \mathrm{s}$, defining a reasonable wind speed regime in which webs are operational.

While all web models perform similarly under moderate global (wind) loading (Fig. 2c), the linear elastic and elastic-perfectly-plastic models respond to targeted force application with a more catastrophic, brittlelike failure that results in significantly increased damage. Defining web damage as percentage of failed (broken) threads, we find that the damage of $2.5 \%$ for the natural silk behaviour increases six-fold to $15 \%$ for the elastic-perfectly-plastic model (Fig. 2a, centre panel). Web performance under local loading is generalized by invoking Quantized Fracture Mechanics (QFM) ${ }^{30}$, a theory applicable to describe the failure mechanisms of discrete structures (such as a spider web) and adapted here to incorporate the material behaviours (SI Section S10). A generalized stress-strain behaviour, where $\sigma \sim \varepsilon^{\kappa}$ ( $\kappa$ is a parameter that defines the nonlinear nature of the stress-strain relationship) treated with QFM reveals that the size of the damaged zone in the proximity to a defect increases for materials that feature a softening behaviour (elastic-perfectly-plastic behaviour), whereas a stiffening material (natural silk) results in a decrease of the damage zone (Fig. 3). This is captured by a scaling law $\Omega(\alpha)=1-s^{2 x}$, defining the structural robustness $\Omega$ as the undamaged fraction of the web after failure. Here $\alpha=\kappa /(\kappa+1)$ reflects the stress-strain response (linear elastic case when $a=1 / 2$; stiffening when $a \rightarrow 1$; softening when $a \rightarrow 0$ ), and $S$ is a system-dependent constant (independent of stress-strain relation). Our simulation results agree with the predictions of QFM (Table S5) and confirm that the relative size of the damage zone is a function of the material stress-strain relation and enhanced by the discreteness of the web (SI Section S10). This phenomenon is duly exemplified in spider webs (Figs. 1-2), where the nonlinear stiffening behaviour (as $\boldsymbol{\alpha} \rightarrow 1$ ) is essential for localizing damage and ensuring that a loaded thread becomes a sacrificial element while the majority of the web remains intact. Given the presumed metabolic effort required by the spider for rebuilding an entire web, localized failure is preferential as it does not compromise the structural integrity of the web (see Fig. 1c) and hence allows it to continue to function for prey capture in spite of the damage.

The remarkable strength, toughness and extensibility of individual spider silk threads are thus not the dominating properties that underpin the excellent structural performance of a spider web. Rather, it is the distinct nonlinear softening and subsequent stiffening of dragline silk that is essential to function, as it results in localization of damage to sacrificial threads in a web subjected to targeted (local) loading while minimizing web deformations under moderate wind (global) loading. Each regime of the nonlinear material behaviour of silk (Fig. 1a) thus plays a key role in defining the overall system response under a variety of environmental settings. Other natural silk threads used to form solid materials such as cocoons, rather than aerial webs, typically display different mechanical responses ${ }^{11}$. Indeed, cocoon silk exhibits elasticperfectly-plastic behaviour not suitable for web construction. The softening behaviour typically seen in 
such silks, combined with a solid material structure rather than a discrete mesh, results in a greater spreading of damage that effectively enhances the system's fracture toughness. This is clearly an advantage for the protective role of cocoons, and reminiscent of other biomaterials where mechanical robustness has been attributed to the formation of large plastic regions ${ }^{2,5}$. The opposite is true for webs, where robustness arises from extreme localization of failure at sacrificial elements, with this behaviour enhanced by the stiffening of threads (Figs. 1a, 2a).

The enhanced mechanical performance of the web relies on the integration of material and structure. We suggest that web design principles might be considered in engineering, where current practice uses sacrificial elements solely to dissipate energy (e.g., impact loading, seismic response). In spider webs, discrete sacrificial elements are instead a means to avoid potentially dangerous system-level loading and mitigate structural damage so that despite the small decrease in spider web load capacity (Fig. 2b), the robustness of the structure overall is greatly enhanced (Fig. 3). This allows a spider to repair rather than rebuild, should failure occur. This marks a shift in structural design by ignoring the requirements for the magnitude of a potential load and allowing local failure to occur, a design stipulation that requires joint consideration of material behaviour and structural architecture.

\section{Methods Summary}

The web consists of an arithmetic spiral ${ }^{7}$ supported by radial threads at regular intervals, constructed from two primary elements, radial threads and spiral threads ${ }^{7}$ (Fig. 1b), and modelled using molecular dynamics procedures. We implement five material behaviours: (i) atomistically derived dragline silk behaviour (parameterized from molecular simulations of dragline silk ${ }^{19,20}$ ), as depicted in Fig 1a; (ii) empirically parameterized dragline silk (from experimental data ${ }^{1}$ ); (iii) empirically parameterized viscid silk (from experimental data ${ }^{1}$ ); (iv) ideal linear elastic behaviour; (v) ideal elastic-perfectly-plastic behaviour, incorporated in three arrangements in Models A, B, and C. We consider two types of application of loading, targeted (local) and global (wind) loading. To characterize the mechanical response of the web under targeted loading, a spring-load is imposed to a small section of the web which is increased until failure is incurred (defined by the failure of loaded threads). Wind loading is applied via a constant drag force applied to all web threads. In situ experiments through simple mechanical assays are applied to an orb web of a common European garden spider. We identify a web in its natural environment and deform radial and spiral threads using a mechanical applicator (a metal wire to load threads). During deformation we control the displacement and monitor images using a digital camera. For theoretical analysis we use Quantized Fracture Mechanics (QFM) ${ }^{30}$, a theory applicable to describe failure of discrete structures such as a spider web and adapted here to incorporate the nonlinear stress-strain behaviour of silk. For a detailed description of the models see Methods and SI).

Full Methods and any associated references are available in the online version of the paper at www.nature.com/nature

1 Gosline, J. M., Guerette, P. A., Ortlepp, C. S. \& Savage, K. N. The mechanical design of spider silks: From fibroin sequence to mechanical function. Journal of Experimental Biology 202, 32953303 (1999).

2 Gao, H., Ji, B., Jäger, I. L., Arzt, E. \& Fratzl, P. Materials become insensitive to flaws at nanoscale: Lessons from nature. P. Natl. Acad. Sci. USA 100, 5597-5600 (2003).

3 Aizenberg, J. et al. Skeleton of Euplectella sp.: Structural hierarchy from the nanoscale to the macroscale. Science 309, 275-278 (2005).

$4 \quad$ Vollrath, F. Spider Webs and Silks. Scientific American 266, 70-76 (1992).

$5 \quad$ Kamat, S., Su, X., Ballarini, R. \& Heuer, A. H. Structural basis for the fracture toughness of the shell of the conch Strombus gigas. Nature 405, 1036-1040 (2000). 
Lefevre, T., Rousseau, M. E. \& Pezolet, M. Protein secondary structure and orientation in silk as revealed by Raman spectromicroscopy. Biophysical Journal 92, 2885-2895 (2007).

Vollrath, F. \& Mohren, W. Spiral geometry in the garden spider's orb web Naturwissenschaften 72, 666-667 (1985).

Vollrath, F. Spider Silk: Evolution and 400 Million Years of Spinning, Waiting, Snagging, and Mating. Nature 466, 319-319 (2010).

Agnarsson, I., Kuntner, M. \& Blackledge, T. A. Bioprospecting Finds the Toughest Biological

Material: Extraordinary Silk from a Giant Riverine Orb Spider. PLoS ONE 5(9), e1 1234 (2010).

$\mathrm{Du}$, N. et al. Design of superior spider silk: From nanostructure to mechanical properties.

Biophysical Journal 91, 4528-4535 (2006).

Shao, Z. Z. \& Vollrath, F. Materials: Surprising strength of silkworm silk. Nature 418, 741-741 (2002).

Omenetto, F. G. \& Kaplan, D. L. New Opportunities for an Ancient Material. Science 329, 528-531 (2010).

Ko, K. K. et al. Engineering properties of spider silk. Advanced Fibers, Plastics, Laminates and Composites 702, 17-23 (2002).

Rammensee, S., Slotta, U., Scheibel, T. \& Bausch, A. R. Assembly mechanism of recombinant spider silk proteins. Proceedings of the National Academy of Sciences of the United States of America 105, 6590-6595 (2008).

Vollrath, F., Holtet, T., Thogersen, H. C. \& Frische, S. Structural organization of spider silk. Proceedings of the Royal Society of London Series B-Biological Sciences 263, 147-151 (1996). Aoyanagi, Y. \& Okumura, K. Simple Model for the Mechanics of Spider Webs. Physical Review Letters 104 (2010).

Ko, F. K. \& Jovicic, J. Modeling of mechanical properties and structural design of spider web. Biomacromolecules 5, 780-785 (2004).

Alam, M. S., Wahab, M. A. \& Jenkins, C. H. Mechanics in naturally compliant structures. Mechanics of Materials 39, 145-160 (2007).

Keten, S., Xu, Z. P., Ihle, B. \& Buehler, M. J. Nanoconfinement controls stiffness, strength and mechanical toughness of beta-sheet crystals in silk. Nature Materials 9, 359-367 (2010).

Keten, S. \& Buehler, M. J. Nanostructure and molecular mechanics of spider dragline silk protein assemblies. Journal of The Royal Society Interface 7(53), 1709-1721 (2010).

Alam, M. S. \& Jenkins, C. H. Damage tolerance in naturally compliant structures. Int J Damage Mech 14, 365-384 (2005).

Foelix, R. F. Biology of spiders. 2nd Ed. (Oxford University Press; Georg Thieme Verlag, 1996). Vollrath, F. Biology of spider silk. International Journal of Biological Macromolecules 24, 81-88 (1999).

Termonia, Y. Molecular Modeling of Spider Silk Elasticity. Macromolecules 27, 7378-7381 (1994).

Vepari, C. \& Kaplan, D. L. Silk as a biomaterial. Progress in Polymer Science 32, 991-1007 (2007).

Swanson, B. O., Blackledge, T. A. \& Hayash, C. Y. Spider capture silk: Performance implications of variation in an exceptional biomaterial. Journal of Experimental Zoology Part a-Ecological Genetics and Physiology 308A, 654-666 (2007).

Swanson, B. O., Anderson, S. P., DiGiovine, C., Ross, R. N. \& Dorsey, J. P. The evolution of complex biomaterial performance: The case of spider silk. Integrative and Comparative Biology 49, 21-31 (2009).

Vollrath, F. \& Selden, P. The role of behavior in the evolution of spiders, silks, and webs. Annual Review of Ecology Evolution and Systematics 38, 819-846 (2007).

Keten, S. \& Buehler, M. J. Atomistic model of the spider silk nanostructure. Applied Physics Letters 96, 153701 (2010). 

(2004).

Supplementary Information is linked to the online version of the paper at www.nature.com/nature.

Acknowledgements This work was supported primarily by the Office of Naval Research (N000141010562) with additional support from the National Science Foundation (MRSEC DMR-0819762, the NSF-REU program, as well as CMMI-0642545) and the Army Research Office (W911NF-09-1-0541 and W911NF-10-1-0127). Support from the MIT-Italy program (MITOR) and a Robert A. Brown Presidential Fellowship is greatly acknowledged. N.M.P. is supported by the METREGEN grant (20092012) "Metrology on a cellular and macromolecular scale for regenerative medicine". An Ideas Starting Grant 2011 BIHSNAM on "Bio-inspired hierarchical super nanomaterials" has been awarded to N.M.P. from the European Research Council, under the European Union's Seventh Framework Programme (FP7/2007-2013) / ERC Grant (agreement number 279985). All simulations have been carried out at MIT's Laboratory for Atomistic and Molecular Mechanics (LAMM). We acknowledge assistance from S. and E. Buehler in taking photographs of the spider web.

Author Contributions S.W.C. and M.J.B. designed the research and analyzed the results. S.W.C. and A.T. developed the material models, performed the simulations, and conducted the simulation data analysis. M.J.B. performed the in situ experiments and analyzed the results. N.P. contributed the theoretical analysis and predictions and analyzed the results. S.W.C., M.J.B., A.T. and N.P. wrote the paper.

Author Information Reprints and permissions information is available atnpg.nature.com/reprints and permissions. The authors declare no competing financial interests. Readers are welcomed to comment on the online version of this article at www.nature.com/nature. Correspondence and requests for materials should be addressed to M.J.B. (하uehler@,MIT.EDU). 


\section{Figure Legends}

Figure 1 | Material behaviour of dragline spider silk, web model, and behaviour of webs under load. a, Derived stress-strain behaviour of dragline silk, parameterized from atomistic simulations and validated against experiments ${ }^{19,20}$. There are four distinct regimes characteristic of $\operatorname{silk}^{19,20}:$ I, Stiff initial response governed by homogeneous stretching; II, Entropic unfolding of semi-amorphous protein domains; III, Stiffening regime as molecules align and load is transferred to the beta-sheet crystals, and; IV, Stick-slip deformation of beta-sheet crystals ${ }^{19}$ until failure. $\mathbf{b}$, Schematic of web model, approximated by a continuous spiral (defined by $d R$ ) supported by eight regular radial silk threads (defined by $d \theta$ ), typical of orb webs ${ }^{7}$. c, Force-displacement curves for loading a defective web (results for Model A; loaded region depicted in red). Case studies include missing spiral segments ( $\mathrm{d} 1$ to $\mathrm{d} 3$ ) and a missing radial thread (d4). Inset: in situ orb web as discovered, containing many defects (marked by green arrows). d, Forcedisplacement behaviour of web, comparing the loading of a single radial thread and a single spiral thread (Model A). e, Loading of a spiral thread results in small web deformation. $\mathbf{f}$, Loading applied at radial threads results in an increase in web deformation. In both cases (e-f) failure is isolated to the pulled thread in simulation and experiment, restricting damage to a small section of the web.

Figure 2 | Web response for varied silk behaviour under targeted (local) and distributed (global) loading. a, Comparison of failure for derived dragline silk, linear elastic, and elastic-perfectly-plastic behaviours (left, Models A, A' and A'). Comparison of failure (centre) confirms localized stresses and minimized damage for the natural nonlinear stiffening silk behaviour. The average stress of each radial thread (bar plots; right) reflects the nonlinear deformation states in the silk. When load is applied locally to a radial thread, other radial threads not subject to applied force reach a stress corresponding to the onset of yielding (i.e., regime II in Fig. 1a). The elastic-perfectly-plastic behaviour leads to an almost homogeneous distribution of stress. b, Force-displacement curves for varying material behaviours (Models A, A' and A" and Model B). c, Web behaviour under distributed (global) wind loading. The plot shows a comparison of the wind-deflection behaviour (Models A, A' and A" and Model B). The initial high stiffness of natural dragline silk enhances structural integrity of the web under such loading. Failure of all webs occurs at wind speeds in excess of $60 \mathrm{~m} / \mathrm{s}$.

\section{Figure 3 | Effects of stress-strain behaviour on structural robustness via Quantized Fracture}

Mechanics. a, Plots of considered stress-strain curves (material behaviour) demonstrating the transition from softening to stiffening behaviours by the nonlinear parameter $\alpha\left(\alpha_{1}=0.3, \alpha_{2}=0.5, \alpha_{3}=0.9\right) . \mathbf{b}$, Structural robustness, $\Omega$, defined as the undamaged fraction of the structure, versus the nonlinear parameter, $\alpha$ (dashed lines indicating $\alpha_{1}, \alpha_{2}, \alpha_{3}$ ), given for three values of the system-dependent constant $S$ $\left(S_{1}=0.1, S_{2}=0.5, S_{3}=0.9\right)$ that captures a range of material properties (such as fracture toughness), system geometry (i.e. crack width or element length), and applied loading conditions. c, Structural robustness $\Omega$, versus system-dependent constant $S$. Universally, the robustness increases with an increase in $\alpha\left(\alpha_{1}<\alpha_{2}<\right.$ $\alpha_{3}$ ), implying that larger nonlinear stiffening results in larger structural robustness, and hence, less damage. 


\section{Methods}

Web geometry. Previous web models have implemented simplified versions of web geometry, most commonly in a concentric circle arrangement ${ }^{16,17,18}$. Here we model a realistic orb web and approximate the it by an arithmetic spiral ${ }^{7}$ defined by the polar equation $\boldsymbol{R}(\theta)=\alpha \cdot \theta$, where spiral spacing is defined by $d R=2 \pi \alpha$, supported by radial threads at regular angular intervals $\left(d \theta=45^{\circ}\right)$. The basic web structure is constructed from two primary silk elements - radial threads and spiral threads ${ }^{7}$, combined with glue-like connections (Fig. 1b). The web is formed by particle-spring elements, with an equilibrium spacing $r_{0}=0.01 \mathrm{~m}$. In Nature, the construction of a stereotypical orb web includes placement of framing threads that act as mooring and a structural foundation for the web ${ }^{7}$. The arrangement of such threads vary according to the anchoring points available to the spider, and exhibit the necessary strength to bear the interior web, and are thus neglected by the current investigation. For a detailed description of the model, see Supplementary Information (SI), Section S2.

Web models. We implement web models based on combinations of five material behaviours: $(i)$, atomistically derived dragline silk (parameterized from molecular simulations of dragline spider silk ${ }^{19,20}$ ) as depicted in Fig 1a; (ii), empirically parameterized dragline silk (from experimental data ${ }^{1}$ ); (iii), empirically parameterized viscid silk (from experimental data ${ }^{1}$ ); (iv), ideal linear elastic behaviour; and (v), ideal elastic-perfectly-plastic behaviour. To explore the differences between the theoretically derived silk models with experimentally measured silks, the materials are implemented in the following web models:

Model A: Atomistically derived dragline silk behaviour used for both the radial and spiral threads, independent of empirical tunings (details SI Section S3.1), to maintain independence from empirical data. Even though such a simple model formulation does not allow us to draw conclusions for phenomena pertaining to specific types of silk, for example, it enables us to understand universal, generic relationships between underlying molecular mechanisms, resulting nonlinear properties of the material, and the failure behaviour of webs. The dragline radial behaviour is used for fitting corresponding linear elastic (Model A', see SI Section S3.3) and elastic-perfectly-plastic (Model A", see SI Section S3.4) models as indicated in Fig. 2a.

Model B: Combination of atomistically derived dragline silk for radial threads (see SI Section S3.1) with empirically parameterized viscid silk behaviour for spiral threads (see SI Section S3.2), to examine the effect of deviations in the stiffness of viscid silk (naturally more compliant than dragline silk). As idealized behaviours (linear elastic or elastic-perfectly-plastic) are parameterized based on the radial response, there are no idealized iterations of Model B.

Model C: A completely empirically parameterized web model, with empirically fitted dragline silk for the radial threads (described in SI Section S3.2) and empirically fitted viscid silk for spiral threads, for a realistic web representation tuned by experimental data ${ }^{1}$. The empirical dragline behaviour is for fitting corresponding linear elastic (Model C', see SI Section S3.3) and elastic-perfectly-plastic (Model C', see SI Section S3.4).

The results of the empirically parameterized model are discussed in SI Section S5. The model framework used here can be easily adapted for other species of spiders, associated silk properties, and web geometries. Using a particle dynamics formulation (motivated by molecular dynamics), the total energy of the web system is defined as:

$$
U_{\text {web }}=\Sigma_{\text {threada }} \phi_{\text {materjal }} \text {, }
$$

for the summation of the elastic potentials of all the silk threads, where $\phi_{\text {materi al }}$ refers to the constitutive energy expression of the specific material. 
Atomistically derived dragline silk. Parameterized from full atomistic simulations of major ampullate (MA) dragline spider silk ${ }^{19,20,31,32}$, unaccounted for in previous web studies ${ }^{16,17,18}$. The constitutive behaviour of dragline silk is formulated as:

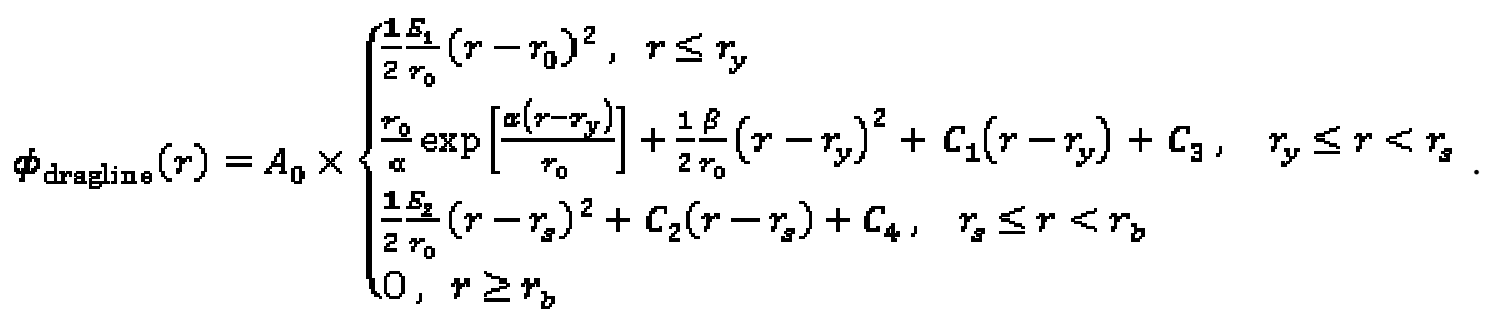

See Table S1 for all parameters.

Empirically parameterized silk. To assess the generality of the results obtained with our atomistically derived behaviour, we implement empirically fitted material behaviours for Models B and $\mathrm{C}^{1}$. The functional form of the empirically parameterized dragline (radial) silk is identical to that of the atomistically derived dragline silk (described by Eq. (2)). To represent the J-shaped viscid silk response measured in experimental studies, a combination linear and exponential function is implemented:

$\phi_{\mathrm{vized}}(r)=A_{0} \times\left(a \cdot r_{0} \cdot \exp \left[\frac{r-r_{0}}{r}\right]+\frac{1}{2} b \cdot r\left(\frac{r-2 r_{0}}{r}\right)+c \cdot r\right)$ for $r<r_{\mathrm{b}}$

We fit the parameters in Eqs. (2) and (3) to experiments on Araneus diadematus ${ }^{1}$ for both dragline and viscid silk. See Table $\mathbf{S 2}$ for all parameters.

Idealized material behaviours. For comparison, motivated by earlier studies ${ }^{33}$, we implement a model that allows us to systematically vary the nature of nonlinear behaviour, allowing cases of ideal linear elastic and ideal elastic-perfectly-plastic (softening), in order to develop general insight. The linear elastic behaviour is governed by:

$$
\phi_{\text {linear }}(r)=\frac{1}{2}\left(\frac{\Omega_{\text {linal }} A_{0}}{r_{0}}\right)\left(r-r_{0}\right)^{2} \text { for } r<r_{\mathrm{b}}
$$

while the elastic-perfectly-plastic behaviour is governed by:

$\phi_{\text {plastic }}(r)=\left\{\begin{array}{cc}\frac{\Sigma_{\text {pla atic }} A_{o}}{2 r_{0}}\left(r-r_{0}\right)^{2} & , r_{0} \leq r<r_{y} \\ \frac{E_{\text {pla tic }} A_{o}}{2 r_{0}}\left(r_{y}-r_{0}\right)^{2}+\frac{\Sigma_{\text {platic }} A_{o}}{r_{0}}\left(r_{y}-r_{0}\right)\left(r-r_{y}\right), & r_{y} \leq r<r_{b}\end{array}\right.$.

Both behaviours are parameterized to reflect either the ultimate stress and strain of atomistically derived dragline silk (in Model A) or the ultimate stress and strain of empirically parameterized dragline silk (in Model C) to provide a comparison between material laws and web performance. See Tables S3 and S4 for all parameters.

Loading conditions. We consider two types of application of loading, targeted (local) and global (wind) loading. To characterize the mechanical response and robustness of the web due to local load, a load is imposed to a small section of the web (see SI Section S4), representing, for example, a small piece of debris. A spring-load is increased until failure is incurred (defined by the failure of all loaded threads). Load is imposed to a small section of the web in the out-of-plane direction, offset from the centre of the web. Proximity to the web centre maximizes the structural resistance of the entire web (as compared to loading the web periphery, for example), while the offset is used to apply the load to a known (chosen) radial thread to ease analysis. Deflection of the web (out-of-plane) and applied force is determined. Work to break individual threads is calculated by numerically integrating the force-displacement curves (see SI Section S6). To characterize the mechanical response under wind load (global), a constant force is applied 
to the entire web structure, derived from the equivalent drag force on a cylindrical wire (see SI Section S8). Loads for equivalent wind speeds of 0.5 to $70 \mathrm{~m} / \mathrm{s}$ are applied (all models fail at $70 \mathrm{~m} / \mathrm{s}$ winds).

In situ experimental studies. Experiments on a physical web are carried out based on mechanical assays applied to an orb web of a common European garden spider. We identify a large spider web in its natural environment and ensure that the spider web is in use by a living spider. We deform radial and spiral threads using a mechanical applicator, a small piece of wire that can effectively be used to pull on small structural features. During mechanical deformation of the web we control the displacement and monitor visual images of the web using a digital camera (results shown in Figs. 1e-f). A black plastic plate is placed behind the web to ensure that the web is clearly visible during the experiment for image acquisition.

Stress distribution. Normalized strain energy distributions are considered for radial threads just prior and immediately after web fracture to calculate the average stress as per Equations (2) to (5) (normalized with respect to maximum strain energy at ultimate failure). Spiral threads are not considered because most of the load (and thus elastic resistance) is carried by the radials in this load interval (see SI Section S9).

Theoretical analysis. We use Quantized Fracture Mechanics (QFM) ${ }^{30}$, a theory applicable to generally describe the failure of a discrete mesh-like structure such as a spider web, and adapted here to incorporate the nonlinear material behaviour of silk using a generalized stress $(\sigma)$-strain $(\varepsilon)$ behaviour $\sigma \sim \varepsilon^{k}$. The relative size of the damage zone after failure is given by

$\varphi(\alpha)=s^{2 \alpha}$,

where $S$ is a system-dependent constant reflective of specific material properties (such as fracture toughness), system geometry, and applied loading conditions. The constant $S$ describes the damage associated with the linear elastic behaviour when $2 \alpha \rightarrow 1$ and therefore $\varphi=S$. The fraction of surviving material after failure defines structural robustness:

$\Omega(\alpha)=1-s^{2 x}$,

The parameter $S$ is determined from the linear elastic response as the reference case, and constant for all variations in the stress-strain behaviour. The three material behaviours studied here (Fig. 2a), characteristic of silk, linear elastic and elastic-perfectly-plastic behaviours, are reduced to general nonlinear stress-strain power laws fitted by a single nonlinearity parameter $(\alpha)$ in the QFM theory (Fig. 3). Details see SI Section S10.

31 Nova, A., Keten, S., Pugno, N. M., Redaelli, A. \& Buehler, M. J. Molecular and Nanostructural Mechanisms of Deformation, Strength and Toughness of Spider Silk Fibrils. Nano Letters 10, 26262634 (2010).

32 van Beek, J. D., Hess, S., Vollrath, F. \& Meier, B. H. The molecular structure of spider dragline silk: Folding and orientation of the protein backbone. Proceedings of the National Academy of Sciences of the United States of America 99, 10266-10271 (2002).

33 Buehler, M. J. \& Gao, H. Dynamical fracture instabilities due to local hyperelasticity at crack tips Nature 439, 307-310 (2006). 